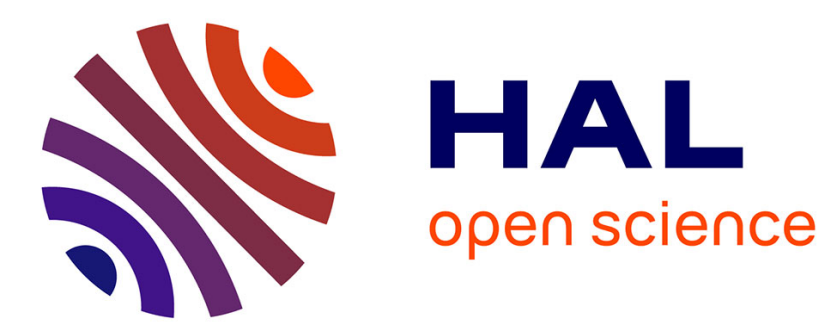

\title{
Improving the sensitivity of amino-silanized sensors using self-structured silane layers: application to fluorescence $\mathrm{pH}$ measurement
}

Rutjaphan Kateklum, Bernard Manuel, Christian Pieralli, Samlee

Mankhetkorn, Bruno Wacogne

\section{To cite this version:}

Rutjaphan Kateklum, Bernard Manuel, Christian Pieralli, Samlee Mankhetkorn, Bruno Wacogne. Improving the sensitivity of amino-silanized sensors using self-structured silane layers: application to fluorescence pH measurement. Sensors and Actuators B: Chemical, 2017, 248, pp.605 - 612. hal02131453

\section{HAL Id: hal-02131453 \\ https://hal.science/hal-02131453}

Submitted on 16 May 2019

HAL is a multi-disciplinary open access archive for the deposit and dissemination of scientific research documents, whether they are published or not. The documents may come from teaching and research institutions in France or abroad, or from public or private research centers.
L'archive ouverte pluridisciplinaire $\mathbf{H A L}$, est destinée au dépôt et à la diffusion de documents scientifiques de niveau recherche, publiés ou non, émanant des établissements d'enseignement et de recherche français ou étrangers, des laboratoires publics ou privés. 


\title{
Improving the sensitivity of amino-silanized sensors using self-structured silane layers: application to fluorescence $\mathrm{pH}$ measurement.
}

Rutjaphan Kateklum ${ }^{1}$, Bernard Gauthier-Manuel ${ }^{1}$, Christian Pieralli ${ }^{1}$, Samlee Mankhetkorn ${ }^{2}$, and Bruno Wacogne ${ }^{1,3}$

${ }^{1}$ Institut FEMTO-ST, CNRS, Univ.Bourgogne Franche-Comté, 25030 Besançon cedex, France.

${ }^{2}$ Center of Excellence in Molecular Imaging, Chiang Mai University, 50200 Chiang Mai, Thailand

${ }^{3}$ INSERM CIC 1431, Besançon University Hospital, 2 place Saint-Jacques, 25000 Besançon, France

Corresponding author: Bruno Wacogne, bruno.wacogne@univ-fcomte.fr

\begin{abstract}
We investigated the possibility to grow molecularly porous amino-silane layers on glass-like substrates. The goal of this work is to show that it is possible to substantially increase the sensitivity of a fluorescence sensor by adjusting the functionalization strategy. Two methods are studied, one using APTMS only and another one using both APTMS and APDMS. We show that, using the second method, sensor sensitivity is improved by a factor of about 5 . In order to demonstrate this, we applied the technique to the grafting of fluorescein in order to build a fluorescence $\mathrm{pH}$ sensor.
\end{abstract}

\section{Abreviations}

SPR, Surface Plasmon Resonance; FTIR, Fourier Transform Infrared spectroscopy; APTMS, (3Aminopropyl)trimethoxysilane; APDMS, (3-Aminopropyl)dimethoxymethylsilane; DMSO, Dimethyl sulfoxide; CFDA-SE, 5(6)-Carboxyuorescein diacetate $\mathrm{N}$-succinimidyl ester.

\section{Keywords}

Amine, FTIR, grafting, fluorescence, $\mathrm{pH}$ sensor

\section{Introduction}

A large number of sensors relies on surface functionalized with ligands which can bind specifically to molecules to be detected. Most of time, amine groups are used to covalently attach ligands onto the surface. These amine groups are generally located at the end of molecules which are grafted onto the sensor surface. Depending on the sensor surface material, different chemistries can be used; thiol chemistry for gold coated surfaces (for SPR experiments for example [1]) or silane chemistry for glass substrates (for ion sensing for example [2]). However, regardless of the type of chemistry used, functionalization often leads to the creation of a monolayer of amine terminated molecules. Therefore, the number of potentially grafted ligands onto the surface remains relatively low.

In the work described here, we focus on amino-silanization and we show how a molecularly porous layer can be formed onto the substrate and how the number of grafted ligands can be 
increased on demand. In order to demonstrate the efficiency of this method, we apply it to $\mathrm{pH}$ measurement using the fluorescence properties of fluorescein molecules covalently grafted to glass-like substrates. Fourier Transform Infrared Spectroscopy (FTIR) is used to assess the number of amine groups grafted onto the substrate. As glass substrate is highly absorbing infrared radiations in the region of interest, we used non-doped silicon substrates, not absorbing for FTIR investigation. The natural silica nanolayer present at the surface of any silicon substrate ensures that functionalization strategy corresponds to the one commonly used for pure glass substrates [3]. Two functionalization methods are compared in these experiments. The first one uses (3-Aminopropyl)trimethoxysilane (APTMS) which produces a layer where only the amine functions at the layer surface are available for dye grafting. The second one uses first APTMS to create the layer grafted on the substrate and second (3Aminopropyl)dimethoxymethylsilane (APDMS) which produces a volume brush-like structure where much more amine functions are available for dye grafting. To the best of our knowledge, such amino-silanization method has not yet been reported.

For both thin monolayer and thick brush-like layer, $\mathrm{pH}$ sensitive fluorescein molecules bearing a succinimidyl ester function are covalently grafted to the amine functions of the silane layer. The fluorescence properties of the surfaces, in particular their $\mathrm{pH}$ dependence, are then investigated.

The next section of the paper presents how substrates should be prepared using either APTMS only or (APTMS+APDMS) methods as well as how fluorescein molecules are covalently attached to the functionalized substrates. Calculation of the amine functions surface concentration based on infrared spectroscopy measurements is also presented. In section 3 , we describe the experimental set-up used in these experiments and we present $\mathrm{pH}$ dependent fluorescence results obtained using both functionalization strategies. Then, a conclusion will be proposed in the last section of this paper.

\section{Surfaces's amino-silanization}

\subsection{Silanization}

The first silanization method uses (3- Aminopropyl)trimethoxysilane (APTMS). This silane exhibits three reactive methoxy functions. The first monolayer uses two of them for grafting on the hydroxylated glass-like surface. The third one remains free for the growth of next reticulated polymer chains. This first method leads to the creation of a compact layer where only surface amine functions (thickness independent) are available for grafting dye molecules. The second method uses first APTMS to create the layer grafted on the substrate and second (3-Aminopropyl)dimethoxymethylsilane (APDMS) with only two reactive functions for the growth of linear polymer chains. This second method leads to the creation of a brush-like layer containing more available amine functions than the first method. Layers formed using both methods are illustrated in figure 1 . Using the second method, the number of available amine functions and thus the potential sensor sensitivity can be adjusted by controlling the functionalization time and therefore the thickness of the brush-like structure. These figures were produced using the "Avogadro" freeware. They do not result from theoretical calculations.

The aminosilane molecular layer is formed by immersion of non-doped silicon fully hydroxylated with 30 min UV-Ozone action into glass vials filled with $1 \%(\mathrm{v} / \mathrm{v}$ ) aminosilane 
solution in anhydrous toluene [4] and a small piece of polypropylene (PP). We noticed that the presence of PP added in the silane solution increases the grafting kinetic of about 10 times. This aspect is not discussed here, but is currently investigated. Probably an additive in PP acts as a catalyst for the silanization reaction. As above mentioned, two silanization methods are compared. The first one where aminosilane is APTMS and the second one where APTMS is used during a short time (15 $\mathrm{min})$ and then APDMS. Full reaction times are equal for both methods.

Substrates are kept in the silanizing solution at $60{ }^{\circ} \mathrm{C}$ for 3 days. They are rinsed with toluene to remove the reagent excess. Washing with dimethyl sulfoxide (DMSO) removes all the polymerized silane molecules which are not chemically linked to the surface. Basic reactions can be summarized by a well-known two steps mechanism:

1: Hydrolysis

$\mathrm{H}_{2} \mathrm{~N}\left(\mathrm{CH}_{2}\right)_{3} \mathrm{Si}\left(\mathrm{OCH}_{3}\right)_{3}+3 \mathrm{H}_{2} \mathrm{O} \rightarrow \mathrm{H}_{2} \mathrm{~N}\left(\mathrm{CH}_{2}\right)_{3} \mathrm{Si}(\mathrm{OH})_{3}+3 \mathrm{CH}_{3} \mathrm{OH}$

\section{2: Condensation}

$-\mathrm{Si}-\mathrm{OH}+-\mathrm{HO}-\mathrm{Si}-\rightarrow-\mathrm{Si}-\mathrm{O}-\mathrm{Si}-+\mathrm{H}_{2} \mathrm{O}$

with $\mathrm{SiOH}$ from hydroxylated silicon surface or hydrolyzed silane molecule.

\subsection{Amine quantification.}

Samples are characterized using Fourier Transform Infrared Spectroscopy (FTIR). FTIR spectra are measured at room temperature using a Perkin-Elmer Frontier spectrometer in transmission mode with a DTGS detector. The spectra are obtained with 10 accumulations and $2 \mathrm{~cm}^{-1}$ resolution.

The absorbance of APTMS was calibrated to obtain a value of the molar attenuation coefficient of a characteristic band of the molecule. For this purpose, a liquid cell, mounted with a silicon window, a barium fluoride one and a $6 \mu \mathrm{m}$ thick mylar spacer is filled with pure APTMS $(c=5.6 \mathrm{M})$. This arrangement presents the advantage that the amplitude of the interference pattern is low enough so that the signal is not disturbed in the measurement zone $\left(2500-3100 \mathrm{~cm}^{-1}\right)$ but large enough to allow a measurement of the true thickness of the film in the $7000-4000 \mathrm{~cm}^{-1}$ frequency range where low absorbance comes from overtones only.

As $\mathrm{NH}_{2}$ bands at 3366 and $3291 \mathrm{~cm}^{-1}$ have low intensity we choose the antisymmetric stretching band $\left(v_{a}^{C H_{2}}\right)$ at $2931 \mathrm{~cm}^{-1}$ of the of the $\mathrm{CH}_{2}$ groups of the propane chain to monitor the grafting. The amount of amine in the grafted layers can be quantified because the number of propane chains is equal to the number of amine functions.

Extraction of this band from FTIR spectra (Figure 2) is conducted via a fit using the LevenbergMarquard algorithm, with a mathematical function composed of five Voigt functions in the range $3700-3100 \mathrm{~cm}^{-1}$. Applying the Beer-Lambert law leads to:

$A_{2931}=\epsilon_{v_{a}}^{C H_{2}}$.c.e

where $A$ is the absorbance, $c$ the concentration in $\mathrm{mol}^{-1}$ and $e$ the thickness of the film. 
As $c$ is known and $e$ is measured, we obtain: $\epsilon_{v_{a}}^{C H_{2}}=254 \mathrm{~mol} . \mathrm{I}^{-1} \cdot \mathrm{cm}^{-1}$.

Then, we proceed in the same manner to extract the amplitudes of the $\mathrm{CH}_{2}$ bands from the measured spectra of grafted samples with the two different chemical processes (figure 3 ).

We can then deduce from these measurements the values of product c.e as the ratio $A_{2931} / \epsilon_{v_{a}}^{C H_{2}}$. We remark that, if we express the concentration in molecule. $\mathrm{nm}^{-3}$ and $e$ in $\mathrm{nm}$, the product c.e has the dimensionality of a number of molecules per $\mathrm{nm}^{2}$. Therefore, the ratio $6.023 \times 10^{6} \mathrm{~A} / \epsilon$ represents the surface concentration in $\mathrm{NH}_{2}$ groups per $\mathrm{nm}^{2}$.

For the two protocols we obtain a similar surface concentration as shown in table 1. However, the number of available amine functions is much higher with the (APTMS+APDMS) method.

\subsection{Sensitive $\mathrm{pH}$ Fluorescein grafting.}

5(6)-Carboxyuorescein diacetate $\mathrm{N}$-succinimidyl ester (CFDA-SE) from Aldrich is a fluorescein molecule which bears two diacetate functions, giving dependence of its fluorescence with $\mathrm{pH}$ and a succinimidyl ester function to allow reactivity with $\mathrm{NH}_{2}$. We use it in solution in anhydrous DMSO at a concentration equals to $2.10^{-3} \mathrm{M}$ to react with aminosilanized silica surfaces according to the reaction described in figure 4 . This process releases $\mathrm{N}$ hydroxysuccinimide (NHS) and leads to the grafting of one dye molecule per accessible $\mathrm{NH}_{2}$ function.

\section{Experimental set-up, results and data analysis.}

\subsection{Experimental set-up.}

The experimental set-up is shown in figure 5. Details concerning suppliers and items codes are indicated in the figure. Schematically, a fibered excitation light source is connected to a fiber reflection probe via an excitation band-filter used to isolate wavelength corresponding to the maximum of the emission spectrum. The fiber reflection probe is composed of 7 multimode fibers, one central and 6 satellites. The central one is used guide excitation light onto the samples, the satellite fibers are used to collect the emitted fluorescence. Fluorescence signals are further filtered using a long-pass filter in order to reject any light coming from the excitation source. Fluorescence spectra are recorded using a micro spectrometer plugged to a computer.

\section{$3.2 \mathrm{pH}$ dependent fluorescence spectra.}

Amino-silanized silicon samples are removed from the oven and cooled down to room temperature. Fluorescein molecules are covalently attached as explained in section 2.3. Buffers with various $\mathrm{pHs}$ are prepared. Fluorescein exhibits a sigmoidal behavior with $\mathrm{pH}$ [5]. Estimating the gain in sensitivity offered by our silanization method only makes sense in the linear region of the sigmoid where fluorescein is interesting for $\mathrm{pH}$ measurement. Therefore, we choose to use $3 \mathrm{pH}$ values in the linear range $(\mathrm{pH}=6.5,7$ and 7.2$)$ plus 1 value at $\mathrm{pH}=8.5$ where the sigmoidal behavior becomes asymptotic.

Drops of buffers (a few $\mu \mathrm{L}$ ) are deposited onto the fluorescein grafted silicon substrates. Because fluorescence spectra are recorded by means of a fiber optic reflection probe, the distance between the substrate and the end-tip of the reflection probe must be kept constant throughout the measurement. Also, buffer drops act as lenses which affect the excitation light incident onto the substrate and the fluorescence emission collection of the satellite fibers of the reflection probe. In order to avoid these experimental difficulties, the same buffer drops 
volume is used for every sample and a glass cover slit must be placed on the substrate after application of the buffer drops. After buffers application, about $10 \mathrm{~min}$ of reaction time is respected in order to reach the chemical equilibrium. $\mathrm{pH}$ dependence of the fluorescence spectra are recorded for both amino-silanization methods. Experimental results are shown in figure 6 .

\subsection{Sensitivity determination.}

All spectra are smoothed using the cubic spline smoothing function in MATLAB ${ }^{\circledR}$ and spectra maxima are automatically computed. Digital signal processing is also used to fit a sigmoid function to the experimental data as shown in figure 7, in accordance with [5]. From this figure, sensitivity of the sensor is computed in the linear range of the sigmoid (from $\mathrm{pH}=6.5$ to $\mathrm{pH}=7.2$ ). A linear regression method leads to the sensitivity of both amino-silanization methods:

- $\mathrm{S}=1278$ Cts per unit $\mathrm{pH}$ for the APDMS method

- $\mathrm{S}=6619$ Cts per unit $\mathrm{pH}$ for the (APDMS+APTMS) method

The gain in sensitivity between the APTMS and the (APDMS+APTMS) methods is 5.18.

\subsection{Signal to noise ratio calculation.}

To do this, noise must be extracted from experimental spectra. A simple way to extract noise is to consider the short wavelength region, below $500 \mathrm{~nm}$ which corresponds to the cut-on wavelength of the emission filter. Figure 8 illustrates noise extraction for $\mathrm{pH}=7$ for the (APDMS+APTMS) methods. Here, noise was centered on zero. The two horizontal lines are an estimation of the noise amplitude, they correspond to twice the standard deviation of the noise.

Now, for both amino-silanization methods, SNR can be numerically calculated. For example, values of the $\mathrm{SNR}$ at $\mathrm{pH}=7$ for both methods are:

- $\mathrm{SNR}=4.2$ for the APDMS method

- $\mathrm{SNR}=21.6$ the (APDMS+APTMS) method

The gain in SNR between the APTMS and the (APDMS+APTMS) methods is 5.14.

Now, if we express the noise amplitude in terms of $\mathrm{pH}$ unit and sensitivity, we can have an idea of the method accuracy. We have:

- $0.24 \mathrm{pH}$ units for the APDMS method

- $0.05 \mathrm{pH}$ units for the (APDMS+APTMS) method

Table 2 summarizes results obtained with both amino-silanization methods at $\mathrm{pH}=7$. This clearly illustrates the improvement provided by the (APTMS+APDMS) method.

\section{Conclusion}

When a high sensitivity is required in molecular sensing, surface functionalization strategy is a key factor. Here, we described a method to create a molecularly porous layer which 
increases the number of available amine functions, hence improving the sensor sensitivity. In the work presented here, we compared two silanization methods, one using APTMS only and another one using both APTMS and APDMS. We showed that, using the second method, sensor sensitivity is improved by a factor of about 5 .

\section{Acknowledgement}

This work was supported by the European Commission [grant number FE2007/2013, operation 36381]. 


\section{References}

[1] M. Bruchard, G. Mignot, V. Derangère, F. Chalmin, A. Chevriaux, F.Végran, W. Boireau, B. Simon, B. Ryffel, J.L. Connat, J. Kanellopoulos, F. Martin, C. Rébé, L. Apetoh, F. Ghiringhelli, Chemotherapy-triggered cathepsin B release in myeloidderived suppressor cells activates the Nlrp3 inammasome and promotes tumor growth, Nat. Med. 19 (2013) 57-64.

[2] O. Meskini, A. Tlili, R. M'ghaïeth, N. Jaffrezic-Renault, A. Abdelghani, Silanization of Porous Silicon Surface for Ion-Sensor Applications, Sen. Lett. 7 (2009) 913-916.

[3] N. Aissaoui, L. Bergaoui, J. Landoulsi, J.F. Lambert, S. Boujday, Silane Layers on Silicon Surfaces: Mechanism of Interaction, Stability, and Influence on Protein Adsorption, Langmuir 28 (2012) 656-665.

[4] Y. Han, D. Mayer, A. Offenhusser, S. Ingebrandt, Surface activation of thin silicon oxides by wet cleaning and silanization, Thin Solid Films 510 (2006) 175-180.

[5] H. Diehl, R. Markuszewski, Studies on fluorescein VII, Talanta 36 (1989) 416-418. 


\section{Figure Caption}

Figure 1: $\quad$ Representation of the silanized layers (Si: pink, C: gray, O: red, N: blue) of a 13mer di (A) and tri (B) silane on a silica surface. ( $\mathrm{H}$ atoms are removed for clarity). (Figures were produced using the "Avogadro" freeware and not issued from theoretical calculations.)

Figure 2: Decomposition of the FTIR (Mes.) in the stretching region of a pure (5.6 M) $6 \mu \mathrm{m}$ thick APTMS film as a sum of Voigt functions. The bottom curve represents the residual of this decomposition.

Figure 3: Extraction of the $\mathrm{CH}_{2} v_{a}$ band (in green) from FTIR measurements of surface grafted with APTMS (A) and APTMS+APDMS (B).

Figure 4: $\quad$ Grafting process of CFDA-SE on aminated surbstrate.

Figure 5: $\quad$ Experimental set-up. Suppliers and part numbers are in italic.

Figure 6: Fluorescence spectra as a function of $\mathrm{pH}$. A: amino-silanization with APTMS only. B: amino-silanization with APTMS+APDMS. Same vertical scale for both graphs.

Figure 7: Extracting the spectra maximum and fitting sigmoid functions.

Figure 8: $\quad$ Noise extraction between 340 and $500 \mathrm{~nm}$. 


\section{Table Caption}

Table 1: Quantification of surface concentration in $\mathrm{NH}_{2}$.

Table 2: $\quad$ Summary of the experimental results at $\mathrm{pH}=7$. 

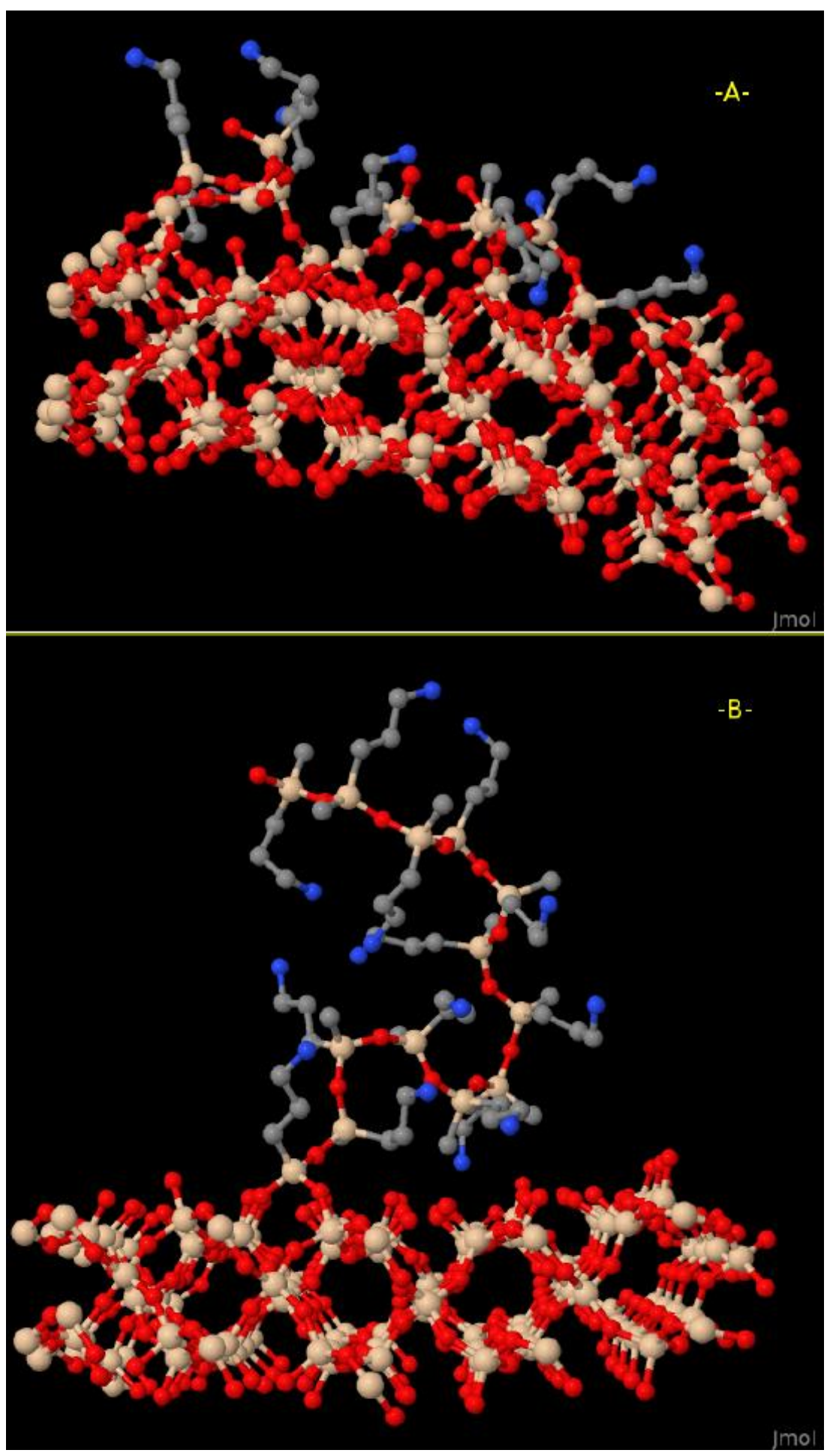

Kateklum, figure 1 


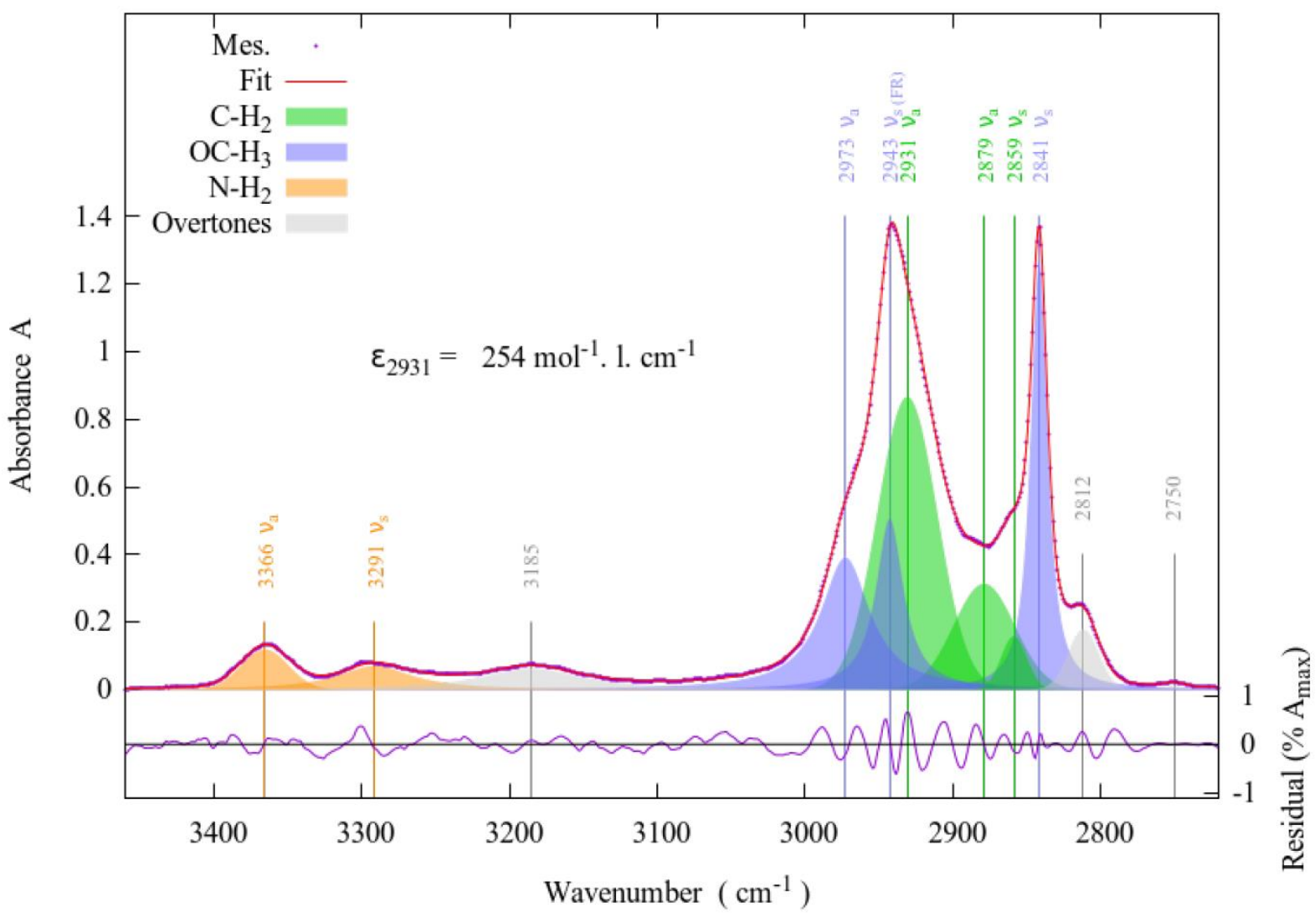

Kateklum, figure 2 

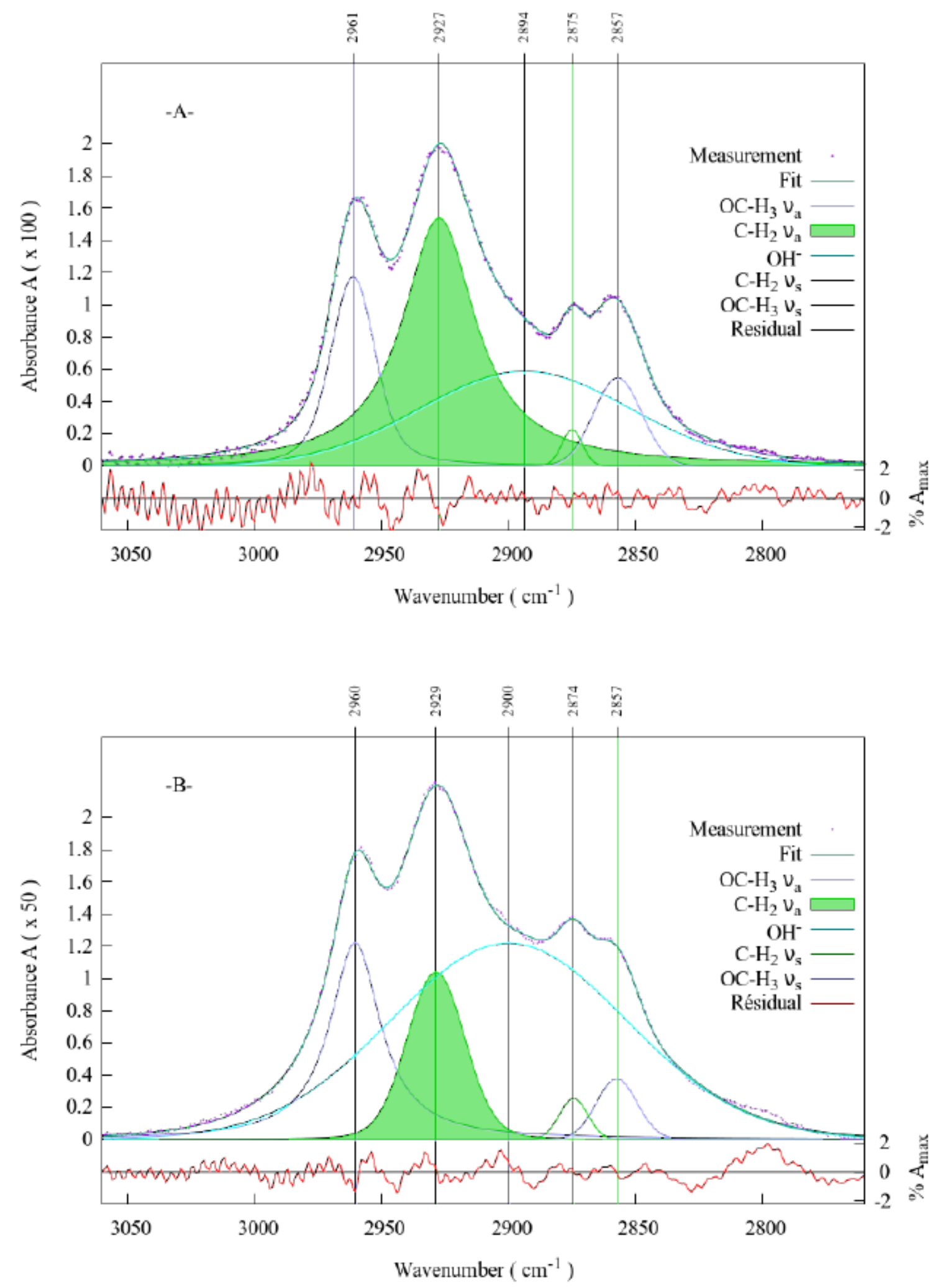

Kateklum, figure 3 
<smiles>CNC1CC(=O)N(OC(=O)Cc2ccccc2)C1=O</smiles>

CFDA-SE
Aminated

Substrate

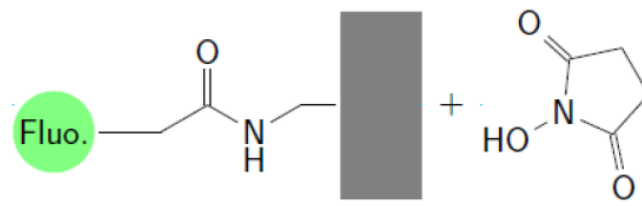

CFDA

grafted

Kateklum, figure 4 


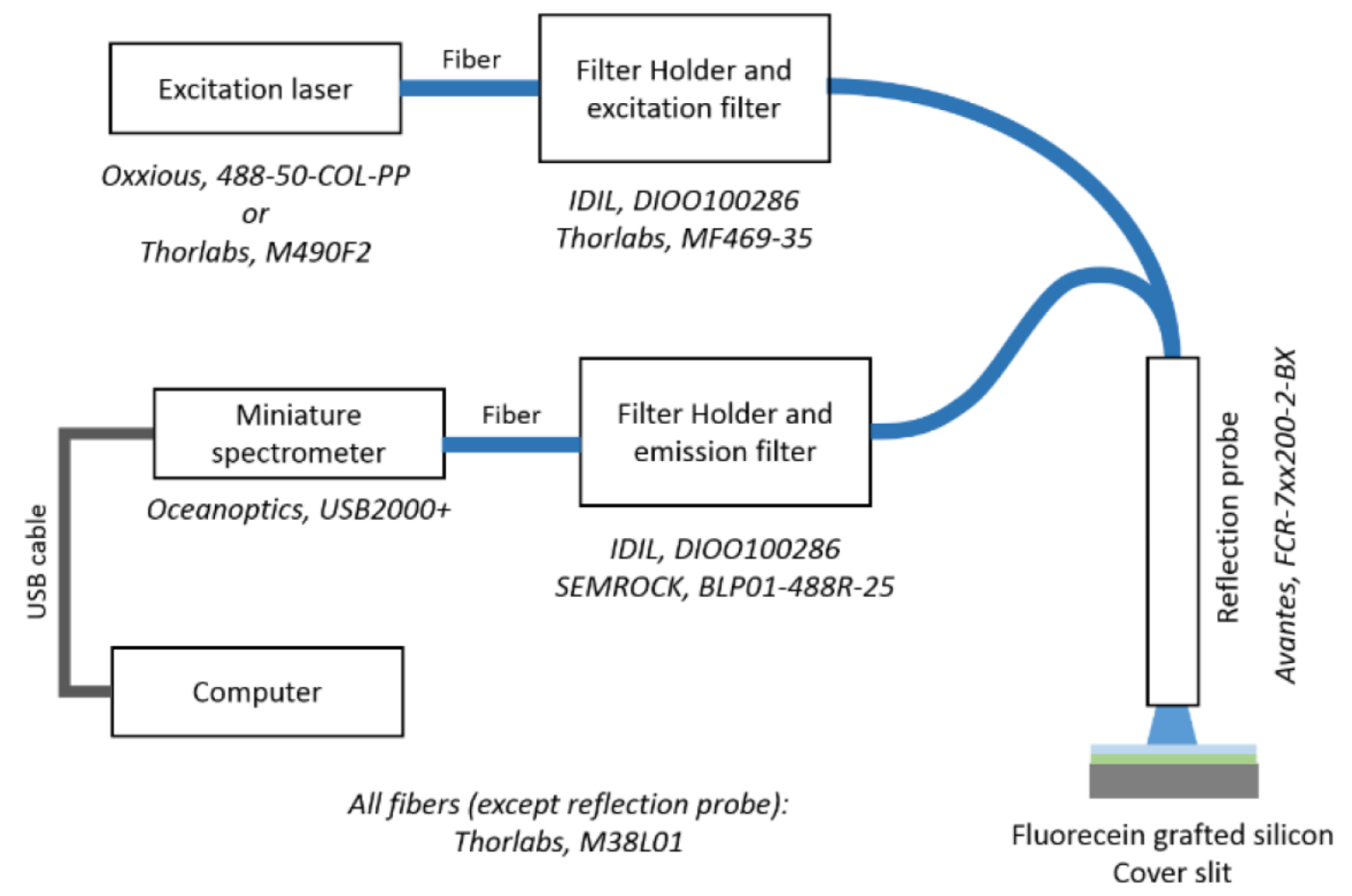

Kateklum, figure 5 

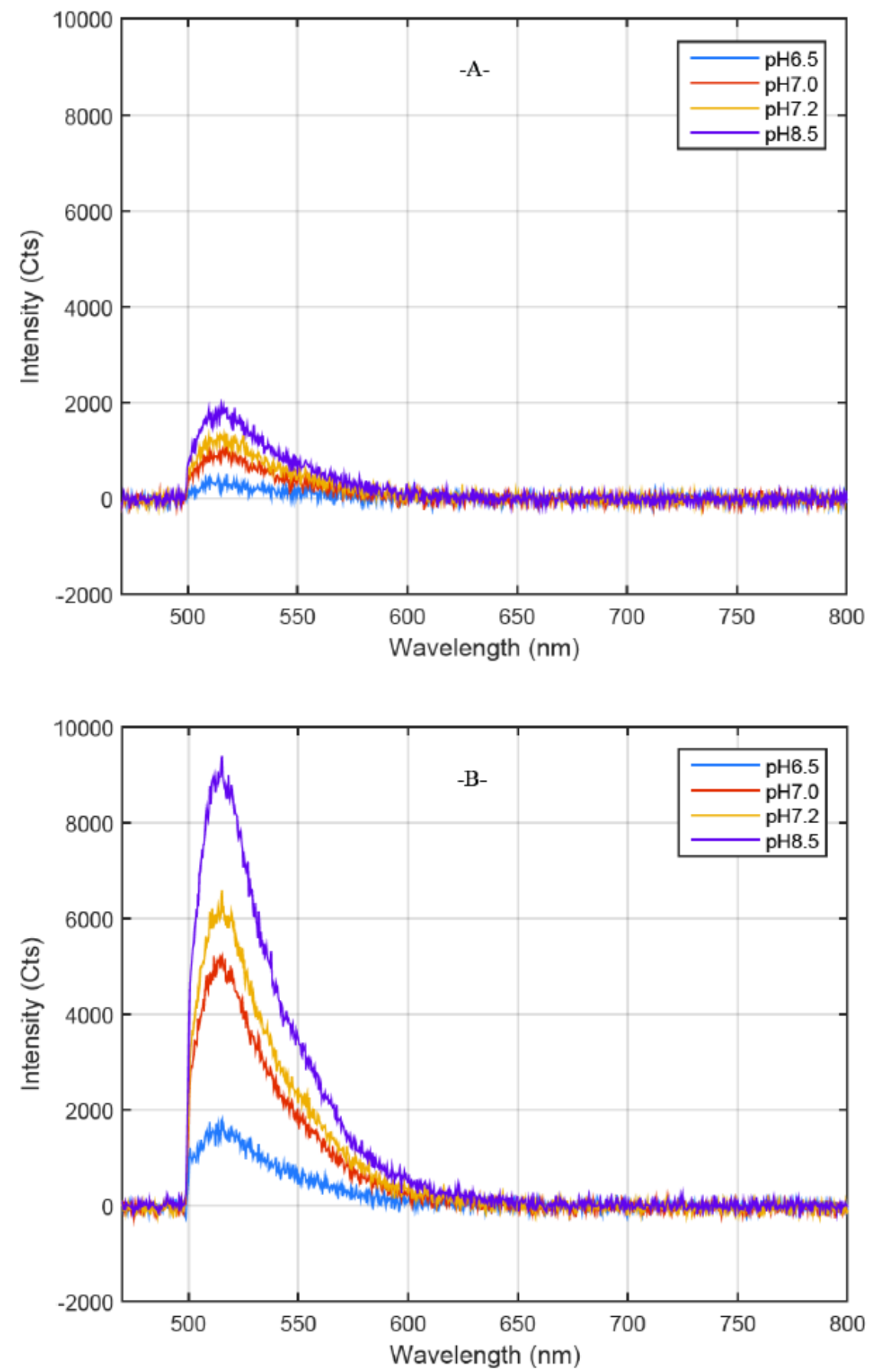

Kateklum, figure 6 


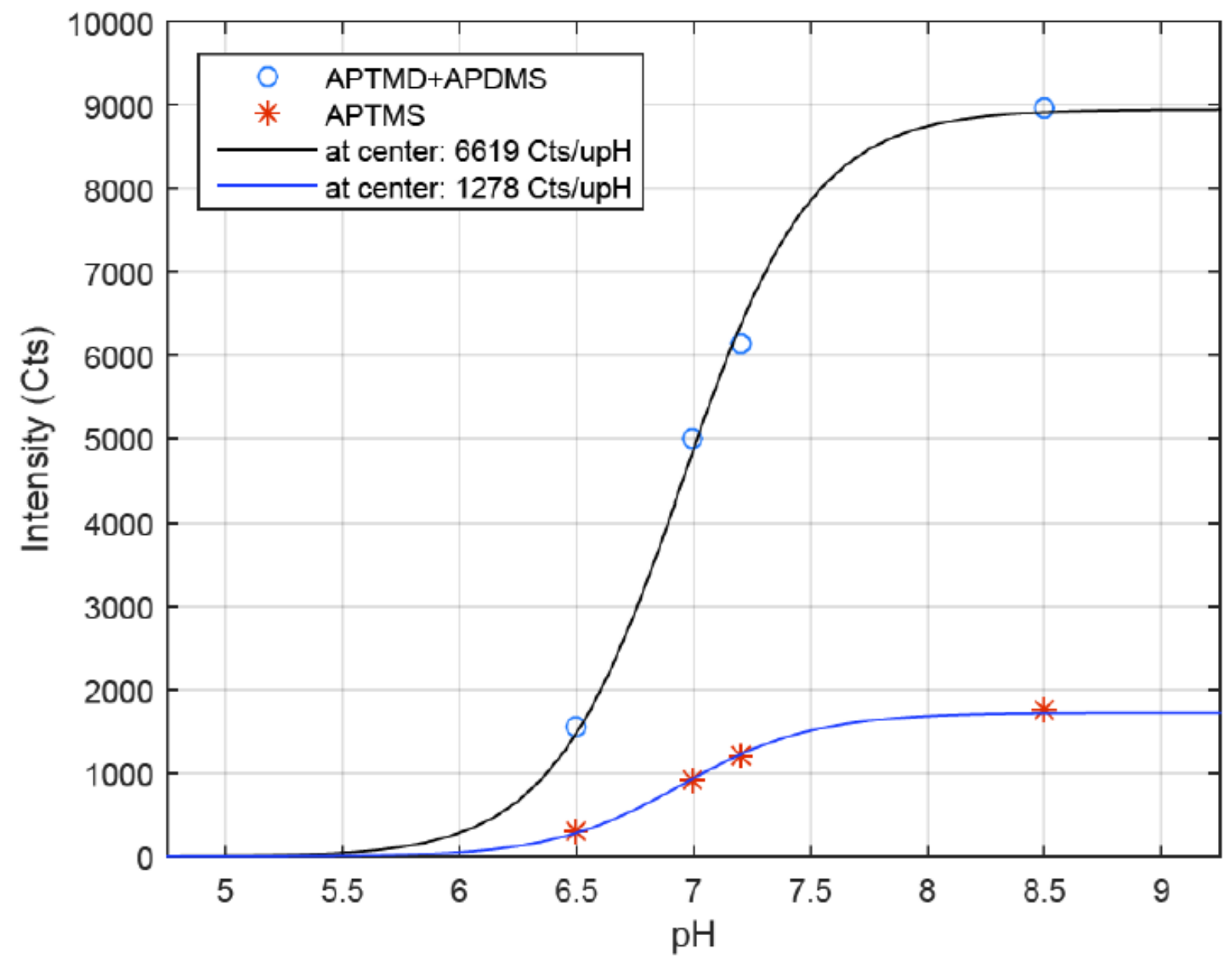

Kateklum, figure 7 


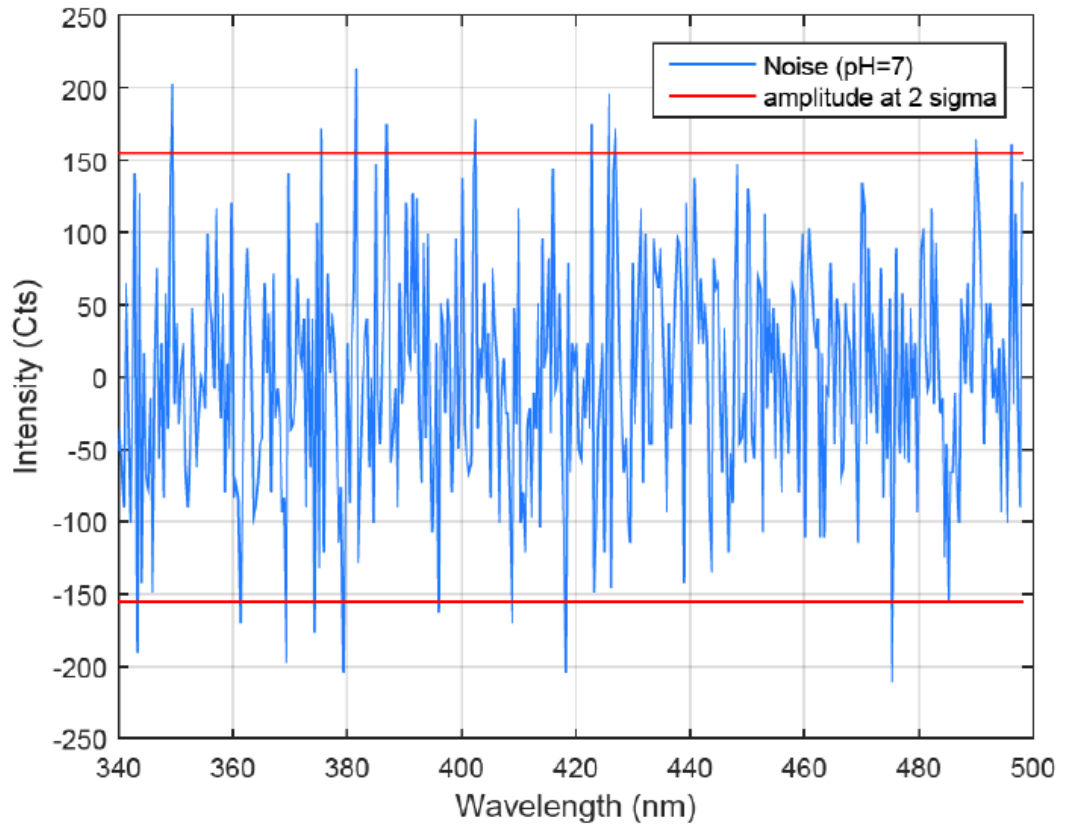

Kateklum, figure 8 